\title{
ANALYSIS OF REACTIVE AND RESISTIVE OPEN WAVEGUIDE FILTERS FOR USE IN MICROWAVE-HEATING APPLICATORS
}

\author{
Francisco J. Clemente-Fernández ${ }^{(\# 1)}$, Juan Monzó-Cabrera ${ }^{(1)}$, Juan L. Pedreño-Molina ${ }^{(1)}$, \\ Antonio J. Lozano-Guerrero ${ }^{(1)}$, and Alejandro Díaz-Morcillo ${ }^{(1)}$ \\ ${ }^{(1)}$ Departamento de Tecnologías de la Información y Comunicaciones, \\ Universidad Politécnica de Cartagena, Murcia, 30202, Spain \\ ${ }^{(\#)}$ francisco.clemente@upct.es
}

\begin{abstract}
In this work the limitations of conventional reactive and resistive filters employed in the open ports of microwave heating applicators are analyzed with CST commercial electromagnetic software. The behavior of simply and doubly corrugated reactive filters is simulated and results are analyzed in order to know the limits of these filters as a function of permittivity of inner materials. Additionally, several configurations for resistive filters implemented with water are assessed.
\end{abstract}

\section{INTRODUCTION}

Microwave energy is widely used in many industrial, scientific and medical applications [1], which usually require the participation of human beings in direct contact with microwave equipment. In particular, industrial microwave ovens often employ open-ended waveguide systems in order to allow a continuous flow of the material to be processed. Thus, safety issues become a main concern in such systems to prevent possible hazardous effects on human tissues due to microwave energy leakage [2].

In general, most works about filters are related to communications bandpass filters dealing with the fundamental mode. Considering waveguide bandstop filters, there are multiple choices such as short-circuited stubs [3], resonant structures [4], [5] or metallic posts [6], to name a few. Focusing on industrial microwave heating, many alternatives to deal with this leakage problem have been proposed in technical literature [7].

One of the most effective alternatives, commonly used in industrial microwave ovens, is based on corrugated reactive filters [8], [9] which reflect back the energy escaped from the applicator simultaneously increasing the efficiency of the process. Although very used in many applications, there are few works that analyze the behavior of these filters as a function of its geometric parameters, such as width or height [10]. Moreover the sample permittivity also affects the final response of the filter, and therefore this influence needs to be studied.

Resistive filters usually use magnetic materials or water in order to eliminate the residual leakage escaping from reactive filtering stages. Also in this case, there are no contributions studying the filtering capabilities of those structures.

In this work the limitations of conventional reactive and resistive filters employed in the open ports of microwave heating applicators are studied with CST commercial electromagnetic software [11]. The behavior of both corrugated and doubly corrugated reactive filters is simulated and results are analyzed in order to know the limits of these filters as a function of permittivity of inner materials. Additionally, several configurations for resistive filters with water are assessed. Optimization procedures based on genetic algorithms have been used by modifying some geometric parameters of the filters in order to obtain the best possible bandstop response. 


\section{OPTIMIZATION TECHNIQUE}

Genetic algorithms (GA) are robust optimization tools based on the concepts of natural selection. A global minimum can be reached in a solution space with weaker minima with the aid of these search procedures. The accuracy and flexibility of genetic algorithms applied to complex non-linear electromagnetic problems have been proved [12].

In this work, genetic algorithms have been employed to modify some geometric parameters of the filter under study in order to center the stopband at $2.45 \mathrm{GHz}$ for all propagating modes, if possible. Thus, an evaluation function $F_{i}$ is defined for each mode $i$ as the sum of differences between a given value of the $S_{21}$ parameter (if greater than $-60 \mathrm{~dB}$ ) and the value of $-60 \mathrm{~dB}$ over a frequency range from 2.4 to $2.5 \mathrm{GHz}$.

$F_{i}=\sum_{j}\left[\left|S_{21}^{i i}\left(f_{j}\right)\right|-(-60 d B)\right], \forall j|| S_{21}^{i i}\left(f_{j}\right) \mid>-60 d B$

where $\left|S_{21}^{i i}\left(f_{j}\right)\right|$ is the magnitude in $\mathrm{dB}$ of the forward transmission coefficient for the mode $i$ with itself and $j$ is the index for each frequency point within the optimized frequency range. The value of $-60 \mathrm{~dB}$ usually ensures a proper filtering of the mode.

The final evaluation function $F$ is defined as the sum of the evaluation functions corresponding to each propagating mode. The GA will try to minimize this function.

$$
F=\sum F_{i}
$$

where $i$ is the index of the considered mode.

All optimization procedures have been carried out by considering vacuum inside the filter. The genetic algorithms tool implemented in Matlab ${ }^{\circledR}$ [13] has been used. Usually 30 generations and 10 individuals per generation have been employed. Good convergence results have been obtained with this configuration, although depending on the number of optimized parameters these configuration parameters might be different.

\section{SIMULATION PROCEDURES}

\section{Reactive corrugated filters}

Regarding the corrugated filters, the manufactured structures studied in [9] have been chosen as a reference. However, these structures are monomode filters dealing with the fundamental mode. In this work the width of these filters has been doubled in order to obtain the performance of the filter when more modes appear. This modification of the width slightly affects the bandstop response of the filter, and therefore it must be improved. In order to do this, the optimization technique based on genetic algorithms has been used by modifying the parameter $d$ in Figure 1 for an empty filter, with no sample inside. In this one and in the following figures which describe the simulated models, it is considered a perfect electric conductor as the background material.

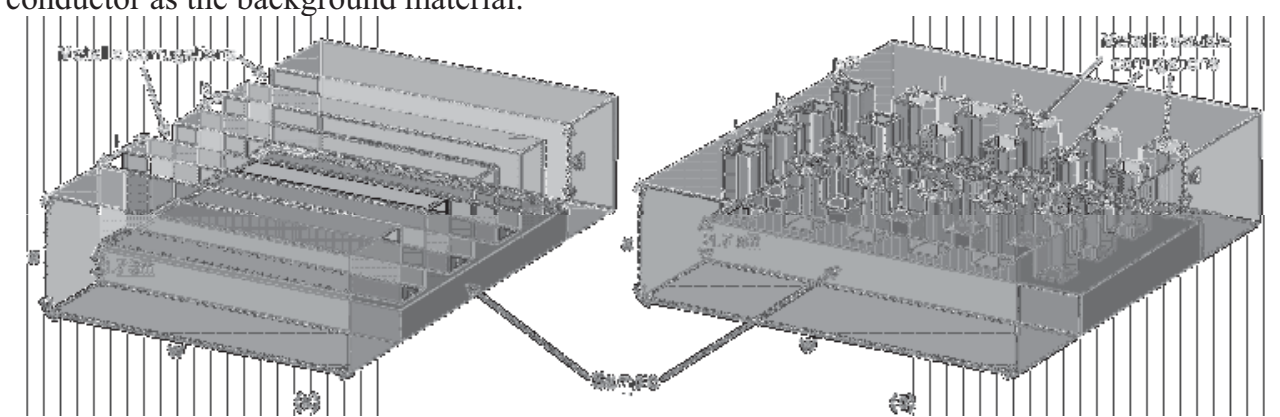

Figure 1. (a) Simply and (b) doubly corrugated filters under study. 
It must be pointed out that these kinds of filters, simply and doubly corrugated, cannot reflect back other modes different from the $\mathrm{TE}_{\mathrm{m} 0}$ ones, since they cannot be decomposed into two TEM wave components [14].

Results have been obtained for three different values of electric permittivity of the sample in order to assess the behavior of the filter when different materials usually treated by microwave energy are placed inside. These values are depicted in Table 1.

Table 1. Dielectric properties of the three employed materials.

\begin{tabular}{l|c}
\hline \multicolumn{2}{c}{ Dielectric Properties } \\
\hline Material 1 & $\varepsilon^{\prime}{ }_{1}=2.55, \tan \delta_{1}=0.0063$ \\
Material 2 & $\varepsilon^{\prime}{ }_{2}=9, \tan \delta_{2}=0.0244$ \\
Material 3 & $\varepsilon^{\prime}{ }_{3}=40, \tan \delta_{3}=0.3$ \\
\hline
\end{tabular}

\section{Resistive Filter with Water Cylinders}

This type of filter is based on the use of cylinders made of a lossy material. In this case, distilled water has been employed, with a dielectric constant of 81 and an electric conductivity of $0.0001 \mathrm{~S} / \mathrm{m}$, as defined in CST database. The design parameters are the separation between cylinders, $l$, the diameter of the cylinders, $D$, and the distance from the top of the filter to the center of the cylinders, $d$, as depicted in Figure 2.

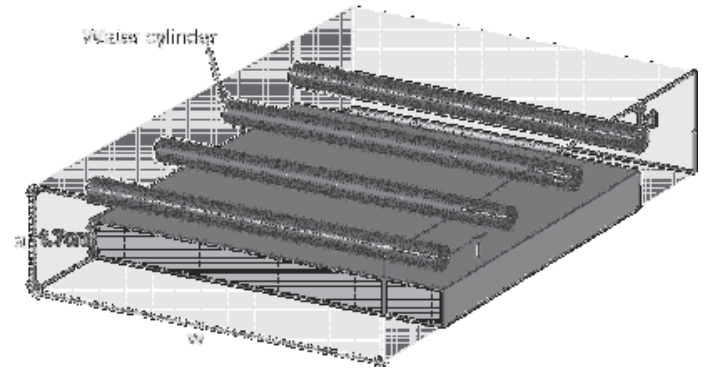

Figure 2. Resistive filter with water cylinders.

An optimization procedure of the empty filter has also been used to find the best configuration by varying $l, d, D$ and the number of cylinders.

\section{Resistive Filter with Water and PTFE Corrugations}

The last simulated configuration is based on a combination of water and PTFE corrugations as depicted in Figure 3. As it can be seen from this figure, several PTFE simple corrugations are filled with distilled water posts.

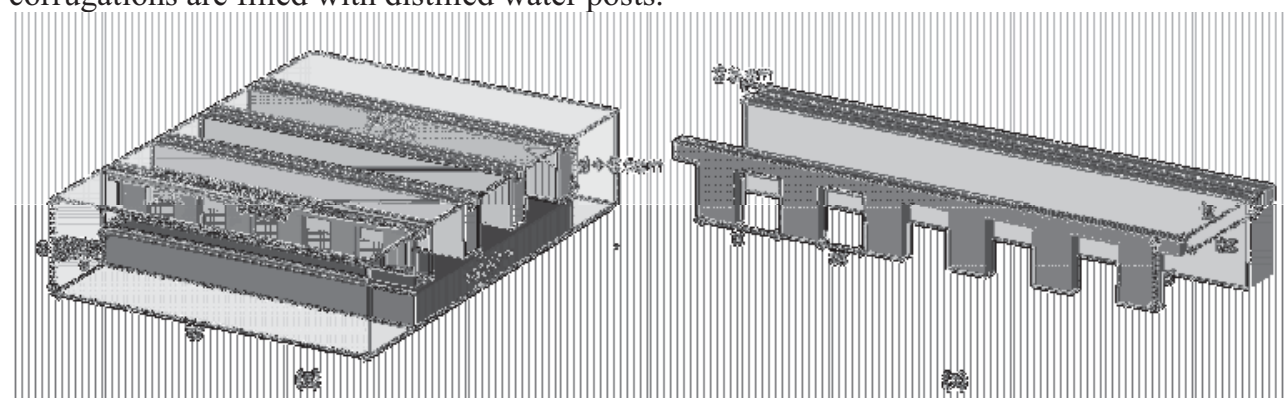

Figure 3. Resistive filter with water and PTFE corrugations. (a) Complete filter and (b) corrugations details. 
In this case the geometric parameters to be optimized are $l z$ and $b z$, which determine the width of the corrugations and the separation between them, and $d$, the length of the water posts.

All the simulations have been made by using the CST time domain solver over a frequency range from 2 to $3 \mathrm{GHz}$.

\section{RESULTS}

Reactive Corrugated Filters

In both, simply and doubly corrugated filters, the evaluation function (2) is minimized by using the geometric parameters shown in Table 2, where the optimized parameters are shown in italics.

Table 2. Geometry of the optimized corrugated filters.

\begin{tabular}{cc|cc}
\hline \multicolumn{2}{c|}{ Simply Corrugated } & \multicolumn{2}{c}{ Doubly } \\
\hline Sections & 3 & Posts & $6 \times 4$ \\
$\mathrm{w}(\mathrm{cm})$ & 17.272 & $\mathrm{w}(\mathrm{cm})$ & 17.272 \\
$\mathrm{a}(\mathrm{cm})$ & 6.164 & $\mathrm{a}(\mathrm{cm})$ & 4.839 \\
$\mathrm{l}(\mathrm{cm})$ & 4.846 & $1(\mathrm{~cm})$ & 2.879 \\
$\mathrm{~b}(\mathrm{~cm})$ & 2.4 & $\mathrm{~b}(\mathrm{~cm})$ & 1.6 \\
$d(\mathrm{~cm})$ & 4.1054 & $d(\mathrm{~cm})$ & 2.7324 \\
\hline
\end{tabular}

In Figures 4 and 5 the magnitude of $S_{21}$ is shown for each material and for the simply and doubly corrugated filter, respectively. In both figures results are depicted for the two modes, $\mathrm{TE}_{10}$ and $\mathrm{TE}_{20}$, which are propagating at $2.45 \mathrm{GHz}$, and different materials.

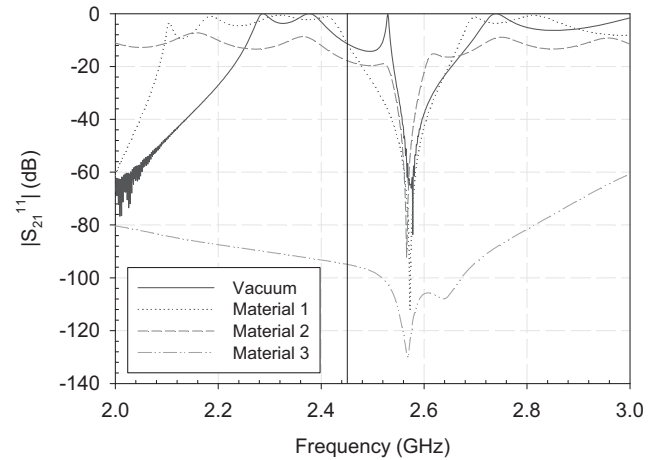

(a)

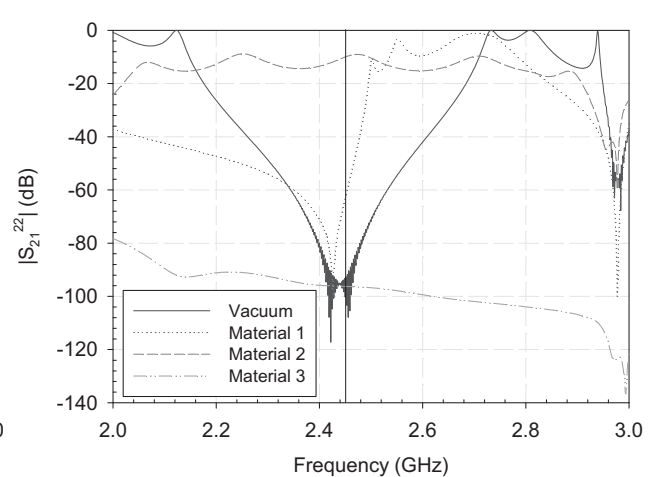

(b)

Figure 4. (a) $S_{21}$ for the $\mathrm{TE}_{10}$ mode and (b) $S_{21}$ for the $\mathrm{TE}_{20}$ mode, for different materials placed inside the simply corrugated filter.

As it can be seen from Figure 4, the simply corrugated filter is able to eliminate only one mode at $2.45 \mathrm{GHz}$, in this case the $\mathrm{TE}_{20}$ mode. It is also possible to filter the $\mathrm{TE}_{10}$ mode by modifying the geometry of the filter, but in that case the $\mathrm{TE}_{20}$ mode would not be properly filtered. In addition, it can also be seen clearly the influence of the electric permittivity of the processed material. These dielectric properties, mainly the dielectric constant, reduce the cutoff frequency of the open port and new modes can propagate and invalidate the protection given by the corrugations. For material 1, the dielectric constant is low enough to not modify significantly the filter response. For material 2, however, the two modes propagate with a 
very low attenuation at $2.45 \mathrm{GHz}$. Finally, for material 3, despite the high dielectric constant, the electric field is attenuated due to high dielectric losses, not because of the filter.

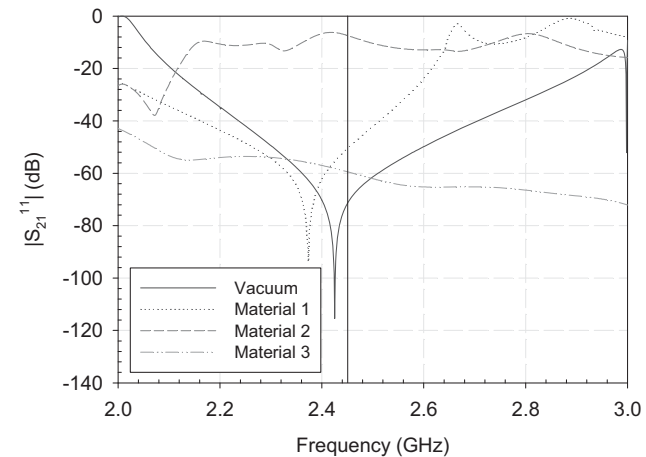

(a)

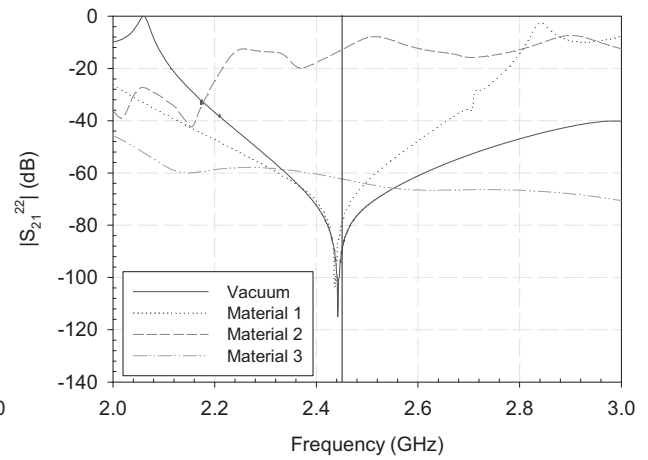

(b)

Figure 5. (a) $S_{21}$ for the $\mathrm{TE}_{10}$ mode and (b) $S_{21}$ for the $\mathrm{TE}_{20}$ mode, for different materials placed inside the doubly corrugated filter.

From Figure 5 it is shown that the doubly corrugated filter is able to deal with more than one $\mathrm{TE}_{\mathrm{m} 0}$ mode. Nevertheless, the influence of the dielectric properties of the sample is similar to the one observed for the simply corrugated filter, because of the reasons explained earlier.

\section{Resistive Filter with Water Cylinders}

First of all, a monomode filter has been simulated in order to obtain the filtering capability of this kind of structures. An optimization procedure has been carried out by varying $l, d, D$ and the number of cylinders. Thus, the used parameters are shown in Table 3, where the optimized parameters are shown in italics. Results for the $\mathrm{TE}_{10}$ mode are shown in Figure 6.

\begin{tabular}{cc}
\hline \multicolumn{2}{c}{ Monomode Filter } \\
\hline Cylinders & 4 \\
$\mathrm{w}(\mathrm{cm})$ & 8.636 \\
$\mathrm{a}(\mathrm{cm})$ & 4.839 \\
$l(\mathrm{~cm})$ & 5.739 \\
$D(\mathrm{~cm})$ & 1.0572 \\
$d(\mathrm{~cm})$ & 1.7043 \\
\hline
\end{tabular}

Table 3. Geometry of the optimized monomode filter with water cylinders.

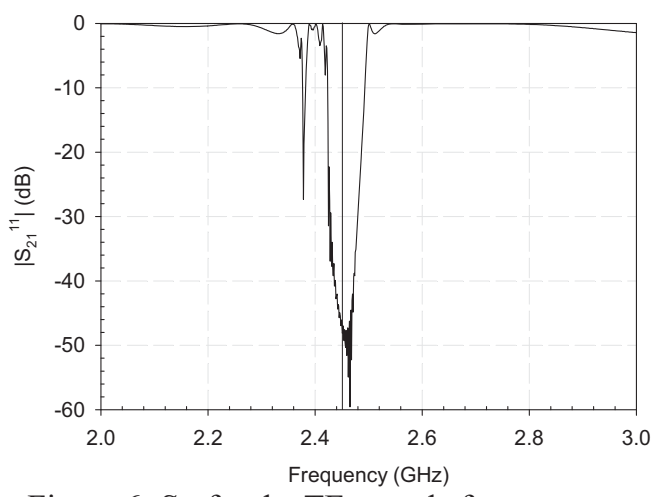

Figure 6. $S_{21}$ for the $\mathrm{TE}_{10}$ mode for an empty filter with water cylinders.

For the monomode filter, it has been possible to obtain a proper bandstop response when no material is placed inside. For a multimode filter, a new optimization procedure has been carried out with a double width empty filter. The optimized parameters are shown in Table 4. Results for this geometry are shown in Figure 7 for each material and for the $\mathrm{TE}_{10}$ (a) and $\mathrm{TE}_{20}$ (b) modes. 


\begin{tabular}{cc}
\hline \multicolumn{2}{c}{ Multimode Filter } \\
\hline Cylinders & 6 \\
$\mathrm{w}(\mathrm{cm})$ & 17.272 \\
$\mathrm{a}(\mathrm{cm})$ & 4.839 \\
$l(\mathrm{~cm})$ & 7.6018 \\
$D(\mathrm{~cm})$ & 1.0529 \\
$d(\mathrm{~cm})$ & 1.5801 \\
\hline
\end{tabular}

Table 4. Geometry of the optimized multimode filter with water cylinders.

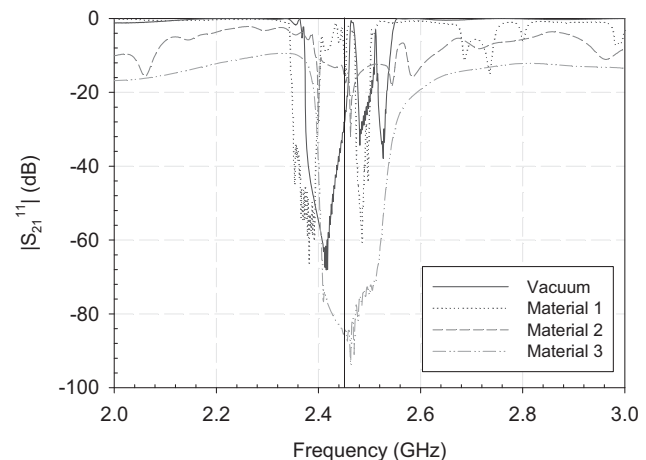

(a)

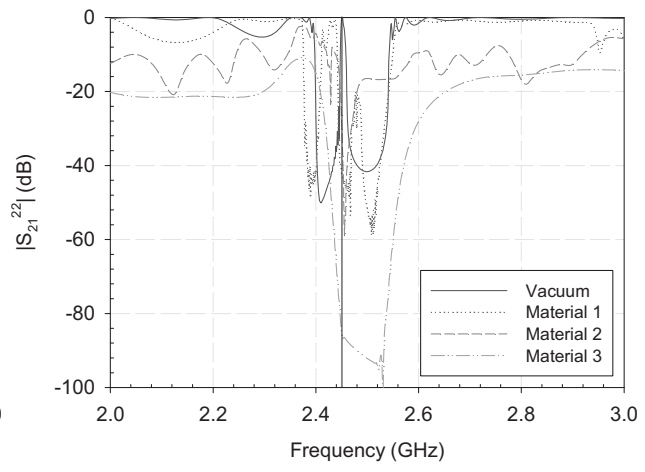

(b)

Figure 7. (a) $S_{21}$ for the $\mathrm{TE}_{10}$ mode and (b) $S_{21}$ for the $\mathrm{TE}_{20}$ mode, for different materials placed inside the filter with water cylinders.

As it can be seen, the filter works well only for the third material, with high dielectric losses. The rest of scenarios are not reliable for a safe usage of the filter.

\section{Resistive Filter with Water and PTFE Corrugations}

In this case, the optimization procedure has been applied to a monomode filter by modifying $l z, b z$ and $d$ from Figure 3 . The used parameters are shown in Table 5, where the optimized parameters are represented in italics. Results for the $\mathrm{TE}_{10}$ mode are shown in Figure 8.

\begin{tabular}{cc}
\hline \multicolumn{2}{c}{ Monomode Filter } \\
\hline Posts & $3 \times 4$ \\
$\mathrm{w}(\mathrm{cm})$ & 8.636 \\
$\mathrm{a}(\mathrm{cm})$ & 4.839 \\
$\mathrm{~lx}(\mathrm{~cm})$ & 2.8787 \\
$\mathrm{bx}(\mathrm{cm})$ & 1.6 \\
$l z(\mathrm{~cm})$ & 5.5006 \\
$b z(\mathrm{~cm})$ & 4.1839 \\
$d(\mathrm{~cm})$ & 1.8783 \\
\hline
\end{tabular}

Table 5. Geometry of the optimized monomode filter with water and PTFE corrugations.

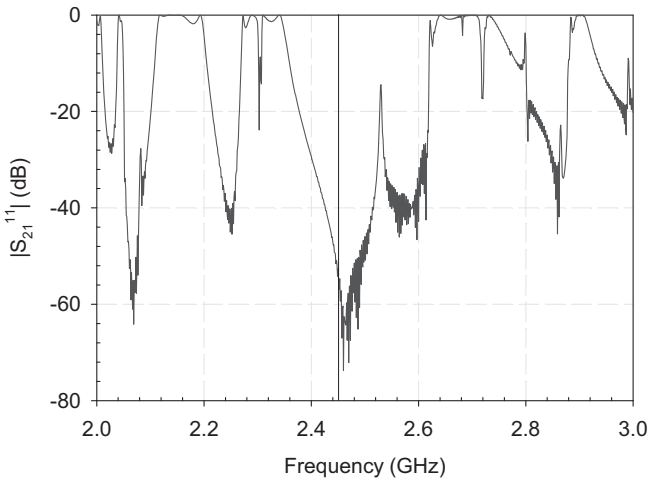

Figure 8. $S_{21}$ for the $\mathrm{TE}_{10}$ mode for an empty filter with water and PTFE corrugations. 
It can be perceived that with this type of filter it is possible to obtain a proper bandstop filter, at least for the fundamental mode and without any material inside the filter.

Once it has been shown that monomode filters can be designed with this type of corrugations, a new optimization procedure has been carried out with a multimode filter. The parameters of the filter used in the simulations are depicted in Table 6 where, again, the optimized parameters are in italics.

Table 6. Geometry of the optimized multimode filter with water and PTFE corrugations.

\begin{tabular}{cc}
\hline \multicolumn{2}{c}{ Multimode Filter } \\
\hline Posts & $6 \times 4$ \\
$\mathrm{w}(\mathrm{cm})$ & 17.272 \\
$\mathrm{a}(\mathrm{cm})$ & 4.839 \\
$\mathrm{~lx}(\mathrm{~cm})$ & 2.8787 \\
$\mathrm{bx}(\mathrm{cm})$ & 1.6 \\
$l z(\mathrm{~cm})$ & 4.6267 \\
$b z(\mathrm{~cm})$ & 4.1373 \\
$d(\mathrm{~cm})$ & 2.3113 \\
\hline
\end{tabular}

Results for this geometry are shown in Figure 9, where the magnitude of $S_{21}$ for each material is represented for the $\mathrm{TE}_{10}(\mathrm{a})$ and $\mathrm{TE}_{20}(\mathrm{~b})$ modes.

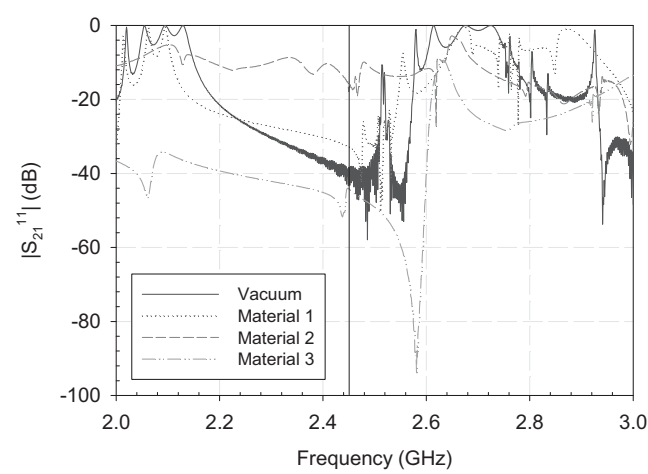

(a)

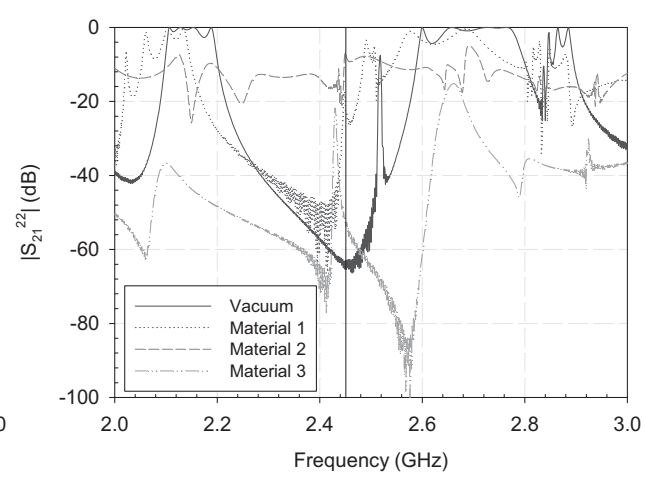

(b)

Figure 8. (a) $S_{21}$ for the $\mathrm{TE}_{10}$ mode and (b) $S_{21}$ for the $\mathrm{TE}_{20}$ mode, for different materials placed inside the filter with water and PTFE corrugations.

In this case, although the response presents sharp variations compared to the one with the metallic corrugations, attenuation levels might be acceptable -if power levels are not very high- at $2.45 \mathrm{GHz}$ for all materials except for material 2, as happened before.

Several optimization procedures have been carried out in order to obtain a proper response when the material 2 is placed inside the studied filters. However, with the analyzed structures it has been impossible to obtain a good solution for this value of dielectric constant.

\section{CONCLUSIONS}

In this work the limitations of conventional reactive and resistive filters employed in the open ports of microwave heating applicators have been studied. In particular, simply and doubly corrugated filters and two different configurations of resistive filters have been analyzed with CST commercial electromagnetic software. 
Results have shown the serious limitations of these filters depending on the electric permittivity of the inner materials. This fact restricts the type of the materials that can be processed, so there is a need of new structures which solve these problems. Therefore, further research is envisaged in that direction.

\section{ACKNOWLEDGEMENTS}

This work was supported in part by the Fundación Séneca, Agencia de Ciencia y Tecnología de la Región de Murcia, under a Séneca predoctoral fellowship with reference 12425/FPI/09 and under the project with reference 11689/PI/09.

\section{REFERENCES}

[1] J. Thuery, Microwaves: Industrial, Scientific and Medical Applications. Norwood, MA: Artech House, 1992.

[2] IEEE Standard for Safety Levels with Respect to Human Exposure to Radio Frequency Electromagnetic Fields, $3 \mathrm{kHz}$ to $300 \mathrm{GHz}, 1992$.

[3] R. Levy, "Compact waveguide bandstop filters for wide stopbands," Microwave Symposium Digest, 2009. MTT '09. IEEE MTT-S International, pp. 1245-1248, Jun. 2009.

[4] C. A. W. Vale, P. Meyer, K. D. Palmer, "A design procedure for bandstop filters in waveguides supporting multiple propagating modes," IEEE Trans. Microw. Theory Tech., vol. 48, no. 12, pp. 2496-2503, Dec. 2000.

[5] P. Meyer, C. A. Vale, W. Steyn, "On the design of waveguide devices using multiple propagating modes," Telecommunications in Modern Satellite, Cable and Broadcasting Service, 2003. TELSIKS 2003. vol.1, pp. 329-336, Oct. 2003.

[6] R. Gesche, N. Lochel, "Two cylindrical obstacles in a rectangular waveguide-resonances and filter applications," IEEE Trans. Microw. Theory Tech., vol. 37, no. 6, pp. 962-968, Jun. 1989.

[7] A. C. Metaxas and R. J. Meredith, Industrial Microwave Heating. Stevenage, U.K.: Peregrinus, 1988

[8] A. L. Vankoughnett and J. G. Dunn, "Doubly corrugated chokes for microwave heating systems," J. Microwave Power, vol. 8, no. 1, pp. 101-110, Jan. 1973.

[9] P. Soto, V. E. Boria, J. M. Catalá-Civera, N. Chouaib, M. Guglielmi, and B. Gimeno, "Analysis, design, and experimental verification of microwave filters for safety issues in open-ended waveguide systems," IEEE Trans. Microw. Theory Tech., vol. 48, no. 11, pp. 2133-2140, Nov. 2000.

[10] J. M. Catalá-Civera, P. Soto, V. E. Boria, J. V. Balbastre, and E. de los Reyes, "Design Parameters of Multiple Reactive Chokes for Open Ports in Microwave Heating Systems," in Advances in Microwave and Radio Frequency Processing. Berlin: Springer, 2006.

[11] http://www.cst.com

[12] Y. Rahmat-Samii and E. Michielsen, Electromagnetic Optimization by Genetic Algorithms. New York: John Wiley \& Sons, 1999.

[13] C. R. Houck, J. A. Joines, and M. G. Kay, "A genetic algorithm for function optimization: A MATLAB implementation," The Mathworks, Natick, MA, NCSU-IE TR 95-09, 1995.

[14] G. Matthaei, L. Young, and E. M. T. Jones, Microwave Filters, Impedance-Matching Networks, and Coupling Structures. Norwood, MA: Artech House, 1980. 